\title{
Immunomodulatory effect of CD200-positive human placenta-derived stem cells in the early phase of stroke
}

\author{
TaeHo Kong ${ }^{1,2,6}$, Ji-Min Park ${ }^{1,2,6}$, Ji Hyon Jang ${ }^{3,6}$, C-Yoon Kim ${ }^{1,4}$, Sang-Hun Bae ${ }^{1,2}$, Yuri Choi ${ }^{1}$, \\ Yun-Hwa Jeong ${ }^{1,2}$, Chul Kim ${ }^{2}$, Sung Woon Chang ${ }^{3}$, Joopyung $\mathrm{Kim}^{5}$ and Jisook Moon ${ }^{1,2}$
}

Human placenta amniotic membrane-derived mesenchymal stem cells (AMSCs) regulate immune responses, and this property can be exploited to treat stroke patients via cell therapy. We investigated the expression profile of AMSCs cultured under hypoxic conditions and observed interesting expression changes in various genes involved in immune regulation. CD200, an antiinflammatory factor and positive regulator of TGF- $\beta$, was more highly expressed under hypoxic conditions than normoxic conditions. Furthermore, AMSCs exhibited inhibition of pro-inflammatory cytokine expression in co-cultures with LPS-primed BV2 microglia, and this effect was decreased in CD200-silenced AMSCs. The AMSCs transplanted into the ischemic rat model of stroke dramatically inhibited the expression of pro-inflammatory cytokines and up-regulated CD200, as compared with the levels in the sham-treated group. Moreover, decreased microglia activation in the boundary region and improvements in behavior were confirmed in AMSC-treated ischemic rats. The results suggested that the highly expressed CD200 from the AMSCs in a hypoxic environment modulates levels of inflammatory cytokines and microglial activation, thus increasing the therapeutic recovery potential after hypoxic-ischemic brain injury, and further demonstrated the immunomodulatory function of AMSCs in a stroke model.

Experimental \& Molecular Medicine (2018) 50, e425; doi:10.1038/emm.2017.233; published online 12 January 2018

\section{INTRODUCTION}

Stroke is an important clinical neurologic disorder and the most common cause of severe adult disability. Brain cells undergo cell death and release their cytoplasmic contents into the extracellular space, thereby evoking inflammatory cascades and amplifying tissue damage. ${ }^{1-3}$ Reactive macrophages and leukocytes are recruited into hypoxia-ischemic brain injury regions. These cells cooperate with resident neurons, astrocytes, and microglia and result in the generation of pro-inflammatory mediators, including enzymes, such as inducible nitric oxide synthase (iNOS) and cyclooxygenase-1 (COX-1), and cytokines and chemokines, such as interleukin-1 (IL-1), IL-6, IL-8, tumor necrosis factor-alpha (TNF- $\alpha$ ) and monocyte chemoattractant protein-1 (MCP-1)., ${ }^{2,4,5}$ Although certain inflammatory conditions may have positive effects on brain repair and neurogenesis. ${ }^{6-8}$, severe bouts of acute or prolonged inflammation in general can decrease neuronal viability and hinder brain regenerative processes under conditions of hypoxia-ischemia brain injury. Previous studies have demonstrated the beneficial effects of neural stem cell and mesenchymal stem cell (MSC) transplantation in central nervous system injuries, including Traumatic Brain Injury (TBI), stroke and spinal cord injury animal models. ${ }^{9-14}$ The major findings of these studies have suggested that MSCs increase host cell survival, improve neurological functional recovery, promote the proliferation of endogenous neural progenitor cells in the neurogenic niche and decrease brain inflammation. ${ }^{15-17}$ Recently, many studies have demonstrated that MSCs possess immunomodulatory properties. MSCs directly inhibit the proliferation of $\mathrm{T}$ lymphocytes and microglial cells and modulate the cytokine secretion profile of dendritic cells and monocytes. ${ }^{18-22}$ The immunosuppressive effect of transplanted MSCs has also been demonstrated in Alzheimer's disease. ${ }^{23,24}$

Global transcriptome analysis in our lab has revealed that AMSCs in a hypoxic environment show increased expression of transforming growth factor-beta (TGF- $\beta$ ), IL-10, IL-4 and

\footnotetext{
${ }^{1}$ Department of Biotechnology, College of Life Science, CHA University, Gyeonggi-do, Korea; ${ }^{2}$ General Research Institute, Bundang CHA general Hospital, Seongnam-si, Korea; ${ }^{3}$ Department of OB. \& GYN., Bundang CHA Medical Center, CHA University, Bundang, Korea; ${ }^{4}$ Department of Laboratory Animal Medicine, College of Veterinary Medicine, Seoul National University, Seoul, Korea and ${ }^{5}$ Department of Neurosurgery, Bundang CHA hospital, CHA university school of medicine, Seongnam-si, Korea

6These authors contributed equally to this work.

Correspondence: Professor J Moon, Department of Biotechnology, College of Life Science, CHA University Pangyo-ro 335 beon-gil, Bundang-gu, Seongnam-si, Gyeonggi-do, Korea.

E-mail: jmoon@cha.ac.kr

Received 7 October 2016; revised 1 April 2017; accepted 7 July 2017
} 

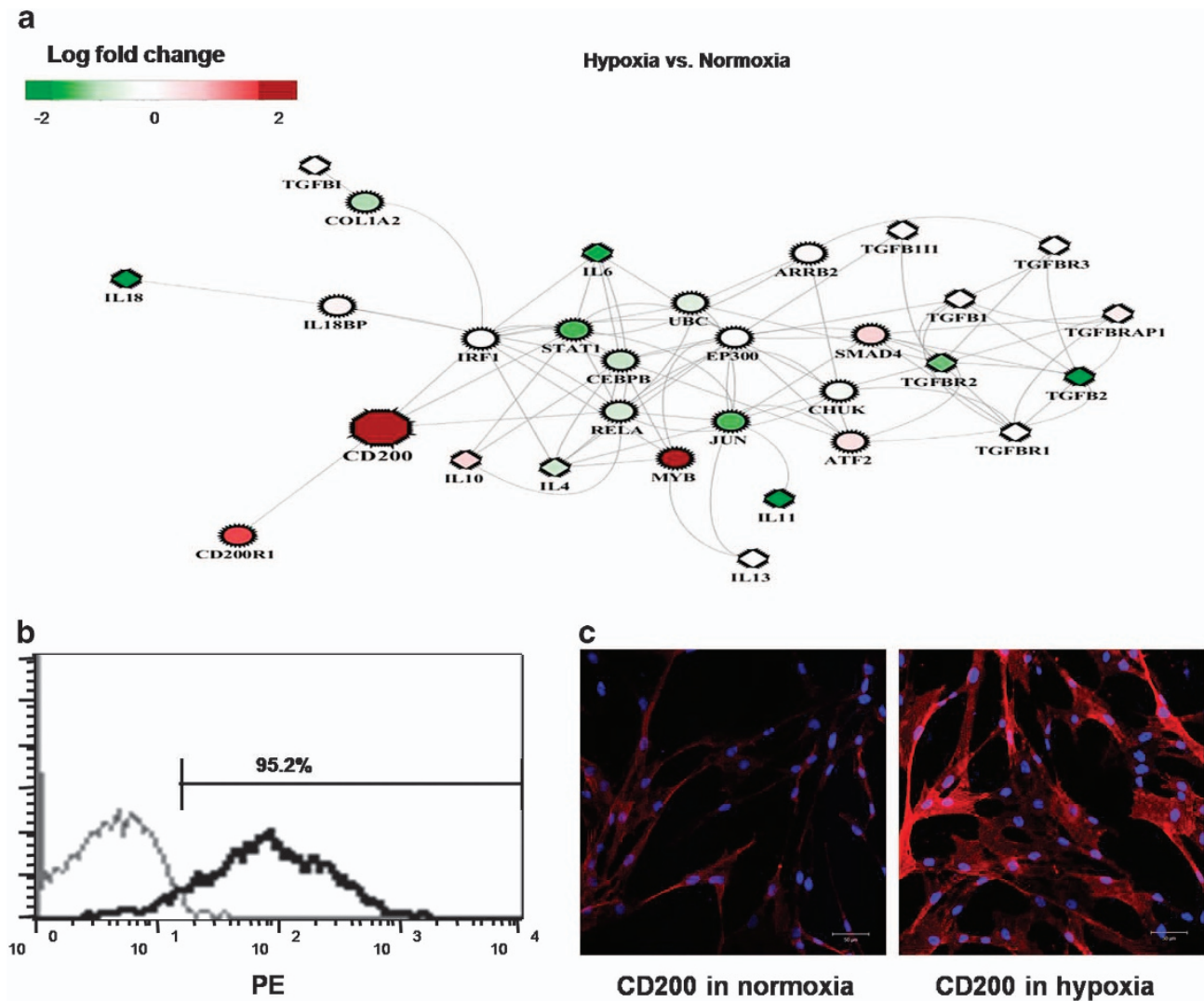

Figure 1 RNA-sequencing analysis and CD200 expression of AMSCs. (a) Network of immune modulation factors in hypoxic compared with normoxic conditions in AMSCs. Log-fold changes are indicated from low (green) to high (red). (b) The strong expression (95.2\%, black line) of CD200 in AMSCs was measured using FACS analysis. (c) Immunocytochemistry detecting CD200 (red) expression in AMSCs with DAPI (blue) under normoxic and hypoxic conditions (scale bar $=25 \mu \mathrm{m}$ ).

CD200. These genes encode anti-inflammation factors predicted to undergo modification, as described above. ${ }^{25}$ Thus, AMSCs behave differently under hypoxic conditions than under normal conditions. Interestingly, CD200, a membrane glycoprotein belonging to the immunoglobulin superfamily, is highly expressed in AMSCs under hypoxic conditions. CD200 is important in the regulation of immune responses and plays a critical role in the maintenance of immune homeostasis. CD200 suppresses the functions of myeloid cells, such as macrophages and neutrophils, by binding to the CD200 receptor and stimulating differentiation into the regulatory $\mathrm{T}$ cell subset. In addition, CD200 facilitates anti-inflammatory TGF- $\beta$ synthesis. ${ }^{26-28}$ However, the functional mechanism of CD200 in neurological disorders and stroke currently remains unclear. We hypothesized that highly expressed CD200 from AMSCs under hypoxic conditions plays a crucial therapeutic role in ischemic stroke rat models by increasing the antiinflammatory response and modulating levels of immune cytokines. In the present study, we demonstrated that ischemic damages gradually increased inflammatory cytokine expression, but CD200-positive AMSCs suppressed the production of proinflammatory cytokines, thus decreasing brain inflammation in a rat stroke model. Finally, we provide preclinical evidence suggesting that $\mathrm{CD} 200$ is a key molecule governing the beneficial effects of AMSCs in stroke patients.

\section{MATERIALS AND METHODS}

\section{AMSC culture and expansion}

All procedures performed in this study were reviewed and approved by the Institutional Review Board of CHA General Hospital, Seoul, Korea. Human placenta was collected immediately after delivery in CHA General Hospital, Seoul, Korea. Informed consent was obtained before the use of patient samples. After the placenta was obtained, the amniotic membrane was carefully dissected and rinsed with phosphate-buffered saline (PBS, Welgene, Gyeongsangbuk-do, Korea). The collected tissue samples were cut into small pieces and digested with $0.5 \%$ collagenase IV (Sigma, St. Louis, MO, USA) in a shaker incubator at $37^{\circ} \mathrm{C}$ for $30 \mathrm{~min}$. The collected cells were cultured in a T-25 flask $\left(2 \times 10^{5}\right.$ cells per $\mathrm{mm}^{2}$, Nunc, Rochester, NY, USA $)$ in alpha-MEM supplemented with $10 \%$ fetal bovine serum (Gibco, Grand Island. NY, USA), $100 \mu \mathrm{g} \mathrm{ml}^{-1}$ each of penicillin and streptomycin (Gibco), $25 \mathrm{ng} \mathrm{ml}^{-1}$ FGF4 (R\&D Systems, Minneapolis, MN, USA), and $1 \mu \mathrm{g} \mathrm{ml}^{-1}$ heparin (Sigma). All cultures were maintained at $37^{\circ} \mathrm{C}$ in an incubator with $5 \% \mathrm{CO}_{2}$, and the culture medium was changed daily. We confirmed the AMSC retained characteristics phenotypes of MSC through flow cytometry analysis (Supplementary Figure S1). In each experiment, the cells were grown to approximately $80 \%$ confluence, and only cells below passage 5 (P5) were used for subsequent experiments. ${ }^{24,29}$

\section{R-seq and network visualization}

Raw read data generated by an Ion Proton sequencer were filtered to exclude poor quality data. Read alignment was performed using STAR 
v.2.3.0 aligner. ${ }^{30}$ on the UNIX platform with default parameters. This analysis produced alignments in the SAM format, which were converted to BAM files and sorted by using SAM tools. ${ }^{31}$ for subsequent analysis. Genome (.fa) and transcript references (.gtf) were obtained from the Ensembl database. The unmapped reads from the first alignment step were re-aligned with bowtie2. ${ }^{32}$ to increase the mapping rate. The HTSeq-count program. ${ }^{33}$ was used to obtain raw gene-level read counts via the intersection-nonempty mode. The genelevel read counts were used as input for DESeq. ${ }^{34}$ to normalize the counts and to analyze differentially expressed genes (DEG). Molecular interactions between CD200 and well-known anti-inflammatory factors were visualized by using Cytoscape. ${ }^{35}$

\section{Immunophenotypic analysis}

Flow cytometry analysis was performed on AMSCs at P5. Briefly, $2 \times 10^{5}$ cells were incubated for $30 \mathrm{~min}$ with fluorescein isothiocyanate (FITC)- or phycoerythrin (PE)-conjugated antibodies directed against the following proteins: SSEA4, TRA-1-60, TRA-1-81, CD34, CD13, CD90, and CD9 (BD, Becton Dickinson, San Jose, CA, USA), and CD200(R\&D system). Ten thousand events were acquired per antibody via fluorescence-aided cell sorting (FACS) by using a Calibur Flow Cytometer. The results were then analyzed using CellQuest Pro software.

\section{siRNA Study}

Transient and stable transfection was performed using Lipofectamine RNAiMAX (Invitrogen, Carlsbad, CA, USA) according to the manufacturer's instructions. Briefly, AMSCs $\left(5.0 \times 10^{4}\right)$ were plated onto 6 -well plates in culture medium containing 10\% FBS without antibiotics at 1 day before transfection, such that the cells were 50 to $60 \%$ confluent at the time of transfection. On the day of transfection, 100 pmol of siRNA was mixed with $10 \mu \mathrm{l}$ of Lipofectamine RNAiMAX in $1 \mathrm{ml}$ of Opti-MEM medium (Invitrogen). The mixture was incubated for $5 \mathrm{~min}$ at room temperature and added to AMSCs in fresh medium lacking serum. siRNA duplex-Lipofectamine RNAiMAX complexes were prepared using either CD200 siRNA (cat No. 1027787, Bioneer, Seoul, Korea). Twenty-four hours after transfection, the cell culture medium was exchanged with fresh medium.

\section{Microglial cell co-culture}

The BV2 mouse microglial cell line was maintained in Dulbecco's modified Eagle's medium supplemented with $10 \%$ fetal bovine serum (Gibco), $2 \mathrm{~mm}$ glutamine, and $100 \mu \mathrm{g} \mathrm{ml}^{-1}$ each of penicillin and streptomycin (Gibco) at $37^{\circ} \mathrm{C}$ in an incubator with $5 \% \mathrm{CO}_{2}$. BV2 cells were stimulated with lipopolysaccharide (LPS) (Sigma), and all experiments were performed in triplicate. For subsequent migration experiments, BV2 cells were cultured in a Transwell system. Transwell (BD) six-well dishes with a membrane pore size of $0.4 \mu \mathrm{m}$ were used. AMSCs $\left(2 \times 10^{5}\right.$ cells per well $)$ were seeded in the Transwell inserts. BV2 cells $\left(2 \times 10^{5}\right.$ cells per well) were seeded in 6-well dishes and incubated overnight to allow attachment. AMSCs and BV2 cells were co-cultured for the LPS stimulation experiments. The plates were treated with $100 \mathrm{ng} / \mathrm{ml}$ of LPS for $12 \mathrm{~h}$.

\section{Nitrite quantification}

The level of $\mathrm{NO}_{2-}$ in the culture supernatants was measured to assess NO production in BV2 cells after LPS treatment. Fifty-microliter aliquots of the samples was mixed with $50 \mu \mathrm{l}$ of Griess reagent (Promega, Madison, WI, USA) in a 96-well plate and incubated at $25^{\circ} \mathrm{C}$ for $10 \mathrm{~min}$. The absorbance was measured at $550 \mathrm{~nm}$ with a microplate reader. $\mathrm{NaNO}_{2}$ was used as the standard to calculate the $\mathrm{NO}_{2}$ - concentration.

\section{MCAO model}

All animal experiments were performed in an authorized animal care facility in accordance with the guidelines of the Committee for the Care and Use of Laboratory Animals. Male Sprague-Dawley rats (8 weeks) were anesthetized with isoflurane (Hana Pharm. Co., Ltd. Seoul, Korea; induction $4 \%$, maintenance $1.5 \%$ ) in $70 \% \mathrm{~N}_{2} \mathrm{O}$ and $30 \% \mathrm{O}_{2}$ using a face mask with a small animal ventilator (Harvard Apparatus). The right external carotid artery was ligated and cut at $5 \mathrm{~mm}$ distal to its bifurcation. A 3-cm length of 4-0 nylon monofilament (AILEE Co., Busan, Korea) was then placed in the external carotid artery. A silk suture (AILEE Co.) was tightened around the monofilament with an intraluminal nylon suture to prevent bleeding, and the micro-clip was removed. The nylon suture was then gently advanced into the internal carotid artery past the circle of Willis until the origin of the middle cerebral artery (MCA) was reached at approximately $2 \mathrm{~cm}$ from the carotid bifurcation. After $2 \mathrm{~h}$ of occlusion, a mini-clip was again placed on the common carotid artery, and the intraluminal suture was removed for MCA reperfusion. After surgery, the animals were randomly assigned into either the sham-control or MCAO treatment group.

\section{Intracerebral transplantation}

We randomly divided all MCAO model rats into two groups: group 1 (control), $10 \mu$ of PBS injection $(n=30)$; and group 2, $10 \mu \mathrm{l}$ of PBS with AMSC $\left(1 \mathrm{X} 10^{5}\right.$ cells) injection $(n=30)$. Twenty-four hours after MCAO model surgery, all rats were anesthetized with a combination mixture of ketamine ( $44 \mathrm{mg} \mathrm{kg}^{-1}$, IP, Huons, Seongnam-si, Korea) and xylazine $\left(13 \mathrm{mg} \mathrm{kg}^{-1}\right.$, IP, Bayer, Seoul, Korea) at a ratio volume of 7 to 3. With a 25-gauge Hamilton syringe, AMSCs were transplanted into ischemic boundary zone (IBZ), right striatum at $\mathrm{AP}=+0.5 \mathrm{~mm} ; \mathrm{ML}=-2.0 \mathrm{~mm} ; \mathrm{DV}=-5.0 \mathrm{~mm}$ according to bregma.

\section{Behavioral tests}

The motor functions of the animals in both groups were evaluated using rotarod tests, and the motor/sensory functions were assessed by an investigator who was blinded to the experimental groups, by using a Modified Neurological Severity Score (mNSS). In the rotarod motor test, the rats were placed on a rotarod cylinder, and the time required for each animal to fall off the device was measured before MCAO and at $1,7,14,21,28$, and 42 days after transplantation. An mNSS test was performed $1,7,14,21,28$, and 42 days after transplantation to measure functional outcome, which was graded on a scale of 0 to 18 (normal score, 0; maximal deficit score, 18). The mNSS test assesses motor, sensory, beam balance, and reflex responses. ${ }^{36}$

\section{Histological analysis of brain tissue}

For histological analysis of the entire damaged zone, the animals were killed under anesthesia, then subjected to intracardial perfusion with paraformaldehyde solution (Samchun Pure Chemical Co., LTD, Gyeonggi-do, Korea) in PBS (4\%, pH 7.4) through the left ventricle at 1 and 6 weeks after transplantation. Brain samples were coronally cryosectioned at a thickness of $30 \mu \mathrm{m}$, and the volume of the damaged tissue was determined at 6 weeks after transplantation. The intact areas of contralateral and ipsilateral hemispheres were calculated in each section by using ImageJ software. The damaged tissue volume was determined by using the following equation: volume (\%)=ipsilateral/ 
contralateral $\times 100$. Immunohistochemical staining was used to examine processes of cell proliferation, microglia/microphage activation, neurogenesis (immature or early neurons), neuron viability and human cell detection. To this end, primary antibodies directed against the following proteins were used: CD200 (1:1000, R\&D system), Iba-1 (1:1000, Wako Chemicals, Osaka, Japan), GFAP (1:1000, Chemicon, Billerica, MA, USA) Doublecortin (DCX; 1:200, Cell Signaling, Beverly, MA, USA), Tuj1 (1:200, Abcam, Cambridge, MA, USA), human nuclei (1:200, Chemicon), NeuN (1:1000, Chemicon). The sections were then incubated with the appropriate secondary antibodies conjugated with Alexa Fluor 488 (1:500, Invitrogen) and Alexa Fluor 594 (1:500, Invitrogen) for 1 hour at room temperature. The images were acquired using a microscope fitted with a digital camera system (Nikon, Japan) and a confocal microscope (Zeiss, Oberkochen, Germany). The images were routed to a Windows PC for quantitative analyses using Adobe Photoshop CS5 software. The total number of the differentiated cells was estimated by using Abercrombie's method. ${ }^{37}$

\section{Quantitative real-time RT-PCR (q-PCR)}

The brains from all groups were isolated, and then the ipsilateral hemispheres were homogenized in liquid nitrogen. Total RNA was extracted from brain tissues, including the graft sites, by using TRIzol reagent (Invitrogen) according to the manufacturer's instructions. We converted $1 \mu \mathrm{g}$ of purified total RNA from the samples into cDNA by using SuperScript III (Invitrogen) and stored all cDNA samples at $-80^{\circ} \mathrm{C}$. Real-time PCR was performed using the SYBR-Green reaction kit (Roche, Indianapolis, IN, USA) according to the manufacturer's instructions in a LightCycler (Roche), and the relative expression of the housekeeping gene GAPDH and TNF- $\alpha$, IFN- $\gamma$, IL-1 $\beta$, IL4, IL6, MCP1, GRO, CD200, IL-10 and TGF- $\beta$ was determined. The primers used for real-time PCR are listed in Supplementary Table S1. The values for target gene expression were normalized against those of GAPDH.

\section{Western blot}

The ipsilateral hemispheres of the brain samples isolated from all groups were homogenized in liquid nitrogen. The homogenized samples were lysed in RIPA buffer (Thermo Scientific, USA) containing one protease inhibitor cocktail tablet (Roche). Whole lysates were normalized using the BCA assay (Thermo Scientific), and $30 \mu \mathrm{g}$ of each lysate was subjected to $10 \%$ SDS-PAGE and transferred to a PVDF membrane (Millipore). The membranes were blocked in TBS (50 mm Tris- $\mathrm{HCl}$, pH 7.6, $150 \mathrm{~mm} \mathrm{NaCl}$,) containing 10\% skim milk (Becton Dickinson) and then probed with each antibody. Immunoreactions were conducted using an ECL Western Blotting system (Millipore) and visualized using Ez-Capture MG (ATTO). The following antibodies were used in these experiments: anti-CD200 (1:1000, R\&D Systems), anti-CD4 (1:200, R\&D Systems), anti-CD25 (1:500, Abbiotec, USA), anti-Foxp3 (1:1000), and anti- $\beta$-actin (1:2000, Santa Cruz, USA).

\section{Statistical analysis}

Statistical analyses were conducted on a CHA University mainframe computer using the Statistical Analysis System (IBM SPSS Korea, Inc., Seoul, Korea), version 22.0. The data are presented as the means \pm SEM. All statistics were calculated using independent $t$-test ( $t$-test) or analysis of variance (ANOVA with a post hoc LSD test). A $P$-value of $<0.05$ was considered significant.

\section{RESULTS}

\section{CD200 is highly expressed in AMSCs under hypoxic conditions}

Hypoxic environments induced by stroke may promote or hinder the therapeutic ability of AMSCs, thus raising the question of how AMSCs remain functionally intact to regulate immune responses under conditions of hypoxic stress. We investigated how AMSCs react to hypoxic conditions by using RNA sequencing. The analysis revealed that various genes involved in immune regulation exhibit interesting expression changes under hypoxic conditions. More importantly, CD200, an anti-inflammatory factor and positive regulator of TGF- $\beta$, was more highly expressed under hypoxic conditions than under normoxic conditions. Assuming that CD200 plays a critical role in inhibiting inflammation by cooperating with other factors, we investigated the potential molecular interactions between CD200 and well-known anti-inflammatory factors in the interactome. ${ }^{38,39}$ by studying the genes exhibiting the shortest distances. Integration of gene expression changes in AMSCs into the interacting genes interestingly revealed that among immune-related factors, only CD200 was significantly up-regulated $(\log 2$ fold change $>2.5 ; P$-value $<0.05$ ) in the hypoxic environment (Figure 1a). This result suggested that CD200 may trigger an anti-inflammatory process in AMSCs in the early stage of stroke characterized by hypoxic environment, thus probably leading to a series of downstream reactions. To confirm the results of the R-seq analysis, we measured CD200 expression in AMSCs. Strong CD200 expression (95.2\%, black line) was detected by using FACS analysis (Figure 1b). Immunocytochemistry detected CD200 expression in AMSCs with DAPI staining under normoxic and hypoxic conditions (Figure 1c). Although most cells expressed CD200 under both normoxic and hypoxic conditions, higher CD200 expression was observed under hypoxic conditions than under normoxic conditions (Figure 1c).

\section{CD200-positive AMSCs regulate immune-modulation factors} We next investigated whether the CD200-positive AMSCs influence immune-suppressive effects in vitro. AMSCs were stimulated with LPS, which mimics inflammation conditions, and were analyzed using q-PCR. As shown in Figure 2, the stimulated AMSCs dramatically increased CD200 expression $(P<0.05)$ (Figure 2a). Immune-modulation factors, including TGF- $\beta \quad(P<0.05)$, IDO $(P<0.05)$, and IL-10 $(P<0.05)$ (Figures $2 \mathrm{~b}-\mathrm{d}$ ), also exhibited increased expression, thus suggesting that AMSCs play an important role in regulating inflammatory conditions. To determine whether the immunomodulatory function of AMSCs is driven by CD200, we used siRNA interference experiments. The siRNA experiments demonstrated that siCD200 efficiently silenced CD200 expression, and this inhibition was maintained after LPS stimulation (Figure 2a). In contrast, TGF- $\beta$, IDO, and IL-10 expression dramatically decreased after CD200 inhibition and exhibited minimal recovery after LPS stimulation (Figures $2 \mathrm{~b}-\mathrm{d}$ ). These results indicated that CD200 may be a key factor for immunemodulation functions in AMSCs. 

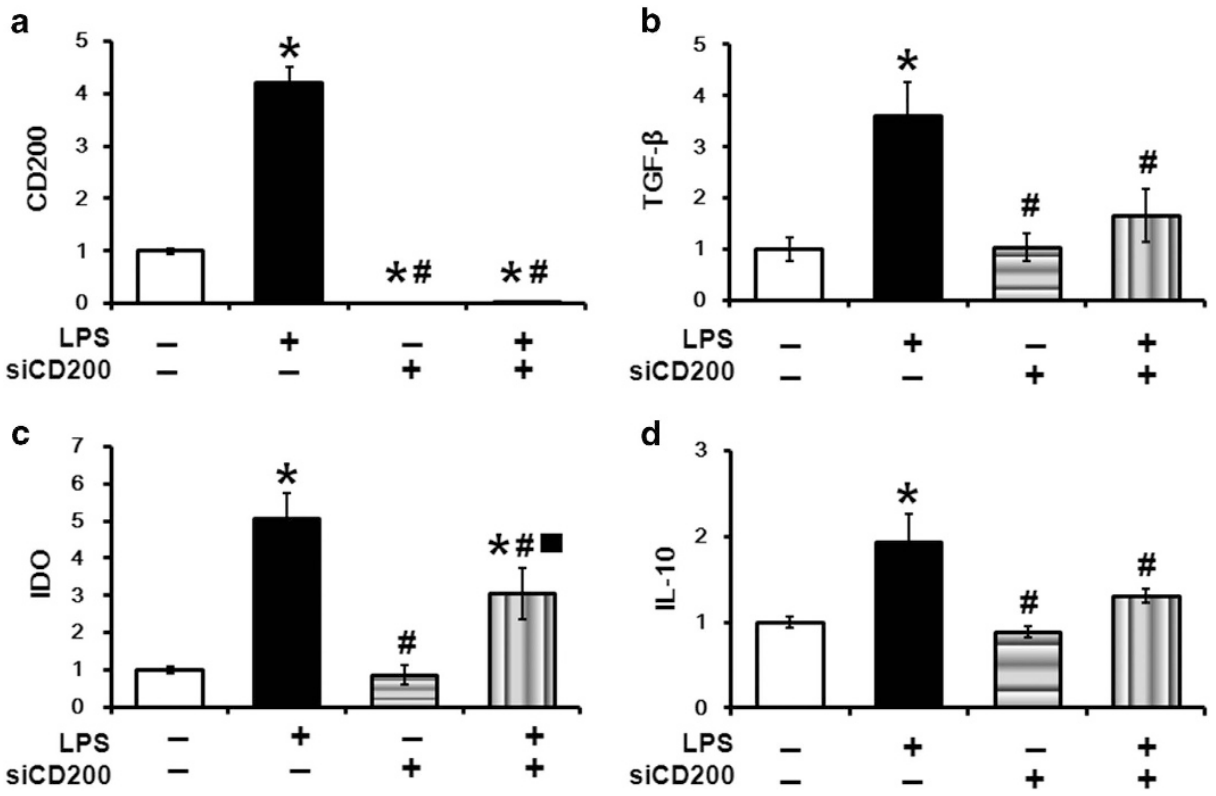

Figure 2 Measurement of immune modulation factors in AMSCs after LPS stimulation and CD200 inhibition. (a) CD200 expression was highly increased after LPS stimulation, as compared with levels in the normal group. CD200 expression was silenced after CD200 siRNA transfection and maintained after LPS stimulation. The expression of immune modulation factors (b) TGF- $\beta$, (c) IDO and (d) IL-10 increased after LPS stimulation. The expression of all factors decreased after CD200 inhibition and showed small range of recovery after LPS stimulation. *: vs Normal; \#: vs LPS; 口: vs CD200 inhibition, $P<0.05$ for each comparison. Data are expressed as the mean standard error of the mean.

CD200-positive AMSCs inhibit LPS-induced microglia cell activation and nitrite oxide production

To confirm the effects of AMSCs in LPS-stimulated BV2 cells, the cells were primed with $100 \mathrm{ng} \mathrm{ml}^{-1}$ LPS and then cocultured with AMSCs. The BV2 cells exhibited morphological changes, including an irregularly rounded and amoeboid shape with cytoplasm distributed over a larger area, wavy edges and clumping. After stimulation with LPS for $12 \mathrm{~h}$, the activated BV2 cells exhibited activated forms. However, the BV2 cells cocultured with AMSCs did not exhibit activation and maintained a small and rounded shape similar to that in the normal group. When stimulated with LPS, BV2 cells were activated, whereas BV2 cell activation was diminished after co-culture with AMSCs. This effect was lost when LPS-induced BV2 cells were co-cultured with AMSCs transfected with CD200 siRNA (Figure 3a). When microglia are activated, they produce potentially neurotoxic substances, such as nitric oxide (NO), oxygen radicals, proteolytic enzymes, and pro-inflammatory cytokines. ${ }^{40}$ We confirmed that the effects of AMSCs on NO production were similar, as shown in Figure 3a. Normal BV2 cells produced minimal nitrite $(7.41 \pm 0.38 \mu \mathrm{M})$. After stimulation with LPS for $12 \mathrm{~h}$, NO production dramatically increased $(33.21 \pm 1.98 \mu \mathrm{M} ; P<0.01)$. In contrast, BV2 cells co-cultured with AMSCs exhibited a significant suppression in nitrite accumulation $(15.74 \pm 2.18 \mu \mathrm{M} ; P<0.01)$. Interestingly, AMSCs transfected with siCD200 exhibited attenuated NO inhibitory effects $(24.66 \pm 2.45 \mu \mathrm{M} ; P<0.05)$ (Figure $3 \mathrm{~b})$. These results suggested that AMSCs suppress microglial activation and associated NO production through CD200.
CD200-positive AMSCs exhibit immune-modulatory effects in LPS-induced activated microglia

To confirm the overall ability of AMSCs to enhance immune modulators in LPS-induced activated microglia, CD200 and TGF- $\beta$ expression in BV2 cells co-cultured with AMSCs in the presence of LPS was measured with q-PCR. We observed significant increases in the immune-modulator CD200 $(P<0.01)$ and TGF- $\beta(P<0.01)$ in these cells compared with controls (Figures $3 c$ and $d$ ). Microglial cells release proinflammatory cytokines, such as IFN- $\gamma$, IL- $1 \beta$, TNF- $\alpha$ and IL-6, after activation. To evaluate the effects of AMSCs on LPSinduced inflammatory cytokine release in BV2 cells, the mRNA expression levels of pro-inflammatory cytokines, including IFN- $\gamma$, IL- $1 \beta$, TNF- $\alpha$, and IL- 6 , were measured in cocultured BV2 cells. As shown in Figures 3e-h, BV2 cells cocultured with AMSCs expressed significantly lower levels of pro-inflammatory genes than did vehicle-treated BV2 cells at 12 hours after stimulation. Furthermore, similar results were observed for the chemokines MCP1 and GRO- $\alpha$ (Figures $3 \mathrm{i}$ and j). To identify the immunomodulatory function of CD200 expressed in AMSCs, we used siRNA interference experiments. Interestingly, AMSCs transfected with siCD200 exhibited attenuated immune-modulation effects on AMSCs (Figure 3). The expression levels of TGF- $\beta$ and other anti-inflammatory factors were dramatically lower in the BV2 cells co-cultured with CD200 siRNA-transfected AMSCs compared with controls (Figure 3). These results suggested that AMSCs suppress microglial cell activation through CD200. Furthermore, similar results were observed in vivo (Supplementary Figure S4c). Altogether, our results indicated that AMSCs induced the up- 

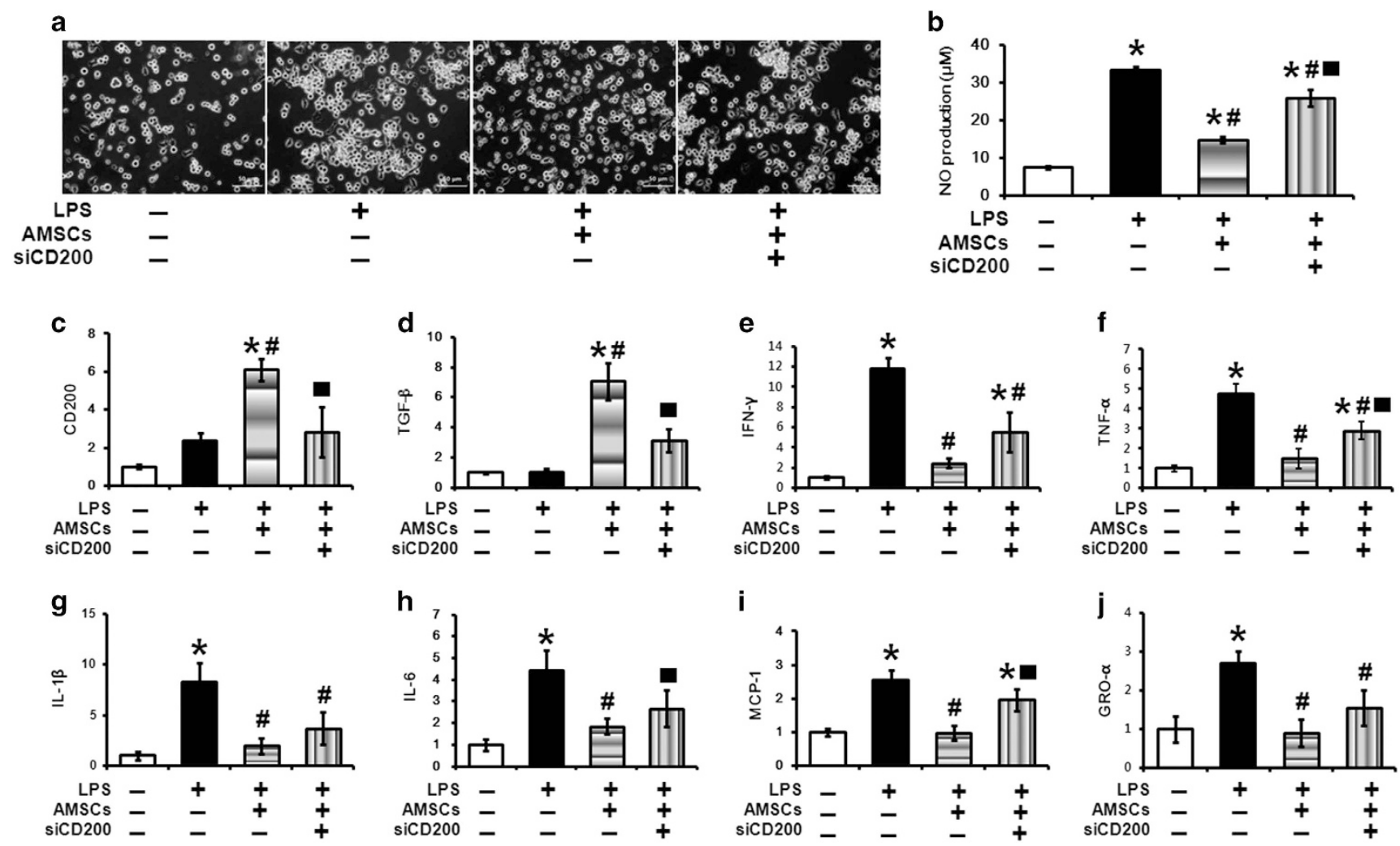

Figure 3 CD200-positive AMSCs inhibit the activation of microglial cells induced by LPS. (a) When stimulated with LPS, BV2 cells were activated (second panel of a), whereas this BV2 cell activation decreased after co-culture with AMSCs (third panel of a). This effect disappeared when LPS-induced BV2 (murine microglial cell line) cells were co-cultured with AMSCs transfected with CD200 siRNA (fourth panel of A). (b) BV2 cells co-cultured with AMSCs exhibited a significant suppression of nitrite accumulation. q-PCR analysis of (c, d) antiinflammatory factors CD200, TGF- $\beta$ and (e-j) pro-inflammatory factors IFN- $\gamma$, TNF- $\alpha$, IL- $1 \beta$, IL-6, MCP- 1 and GRO- $\alpha$ in the same groups above. *: vs Normal; \#: vs LPS; $\square$ : vs LPS + co-culture, $P<0.05$ for each comparison. Data are expressed as the mean standard error of the mean.

regulation of anti-inflammatory factors, including CD200 and TFG- $\beta$, whereas pro-inflammatory cytokines/chemokines were decreased in toxic and stressful environments, thus suggesting that AMSCs improve the compromised immune system.

Survival and migration of CD200-positive AMSCs after intrastriatal transplantation

The rats received an intracranial transplantation of AMSCs at $24 \mathrm{~h}$ after MCAO. With an antibody against human nuclei, AMSCs were clearly detected in the transplanted area at 1 week after transplantation. Human nuclei-positive cells were primarily concentrated at the injected site. Human nuclei-positive cells were detected in the striatum close to the injection site but not in contralateral site at 1 week after cell transplantation (Supplementary Figure S2A-C). In addition, a small proportion of human-nuclei positive cells were co-stained with neural markers, DCX and Tuj1 (3.9\% of cells co-labeled with humannuclei, DCX, and Tuj1) (Supplementary Figure S2D-F). These findings suggested that the transplanted AMSCs differentiated into neurons at 1 week after transplantation.
Immunomodulatory effects of CD200-positive AMSCs in ischemic stroke brain tissue

The most studied cytokines related to inflammation in acute ischemic stroke are TNF- $\alpha$, IFN- $\gamma$, IL- $1 \beta$, IL-10 and TGF- $\beta$. In a previous study, we have demonstrated that CD200 activates anti-inflammatory pathways and induces immune tolerance. To evaluate the immunomodulatory effects of AMSCs on ischemic stroke brain tissue, we analyzed the mRNA expression of pro-inflammatory and anti-inflammatory factors at 1 day and 1 week after transplantation. The injected AMSCs exhibited significantly increased levels of anti-inflammatory factors, including CD200 $(P<0.05)$, IDO $(P<0.05)$ and IL-10 $(P<0.01)$ (Figures $4 \mathrm{a}, \mathrm{b}$ and $\mathrm{d})$. The TGF- $\beta$ expression level was significantly decreased from 1 day to 1 week in the control group, whereas the TGF- $\beta$ expression in the AMSC group persisted for 1 week (Figure 4c). Furthermore, the mRNA expression of pro-inflammatory factors increased in the control group from 1 day to 1 week, however the AMSC group showed only slight changes and in contrast to the control group 1 week after transplantation $((\mathrm{IFN}-\gamma, \quad(P<0.05), \quad$ TNF- $\alpha$ $(P<0.05)$, IL-1 $\beta(P<0.05)$, MCP1 $(P<0.01)$ and GRO- $\alpha))$ (Figures $4 \mathrm{e}-\mathrm{i}$ ). These findings suggested that the injected 
a
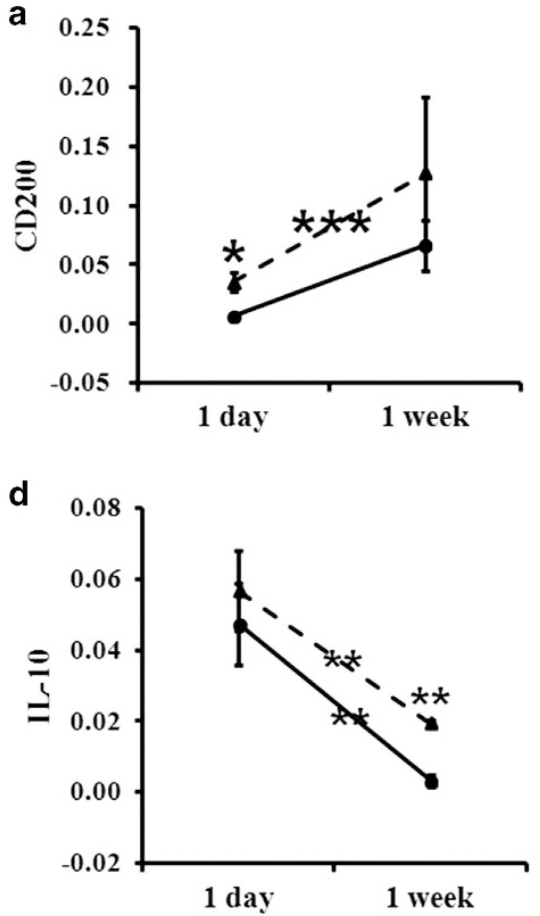

g

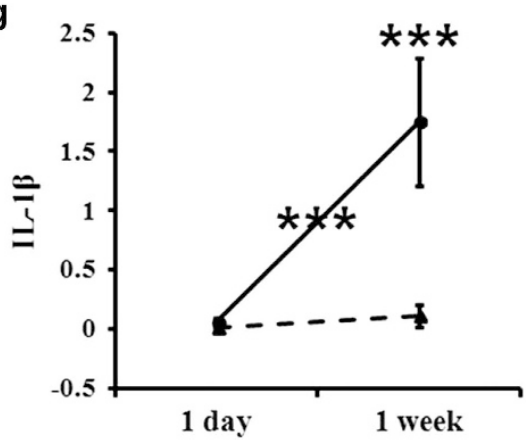

b

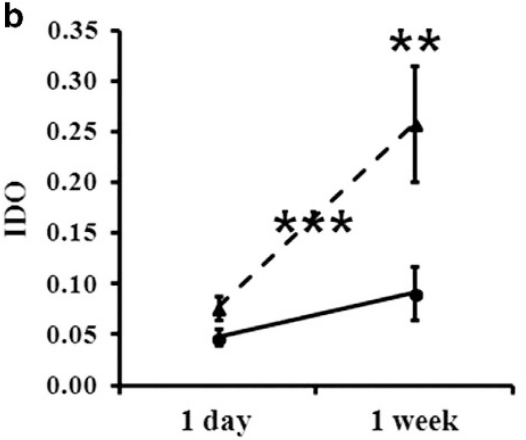

e

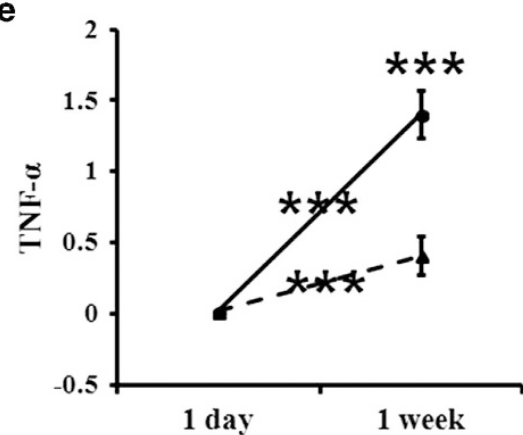

h

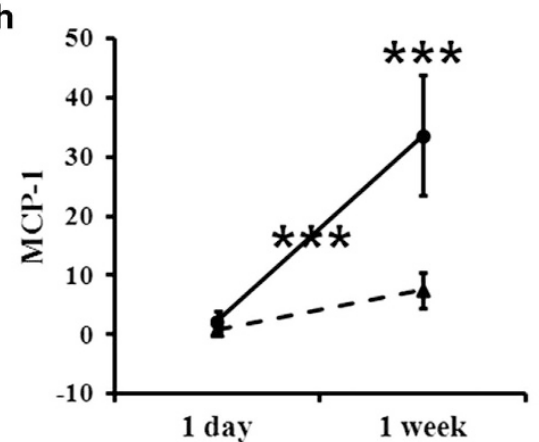

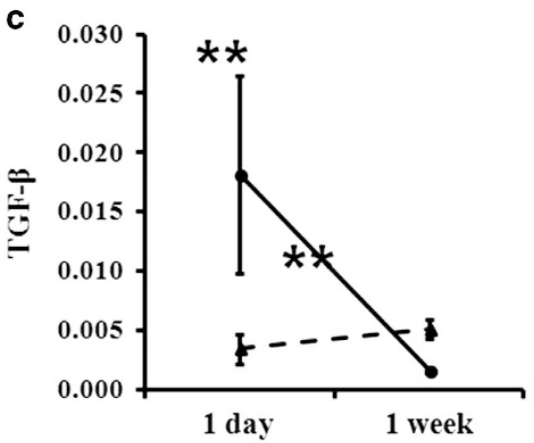

f
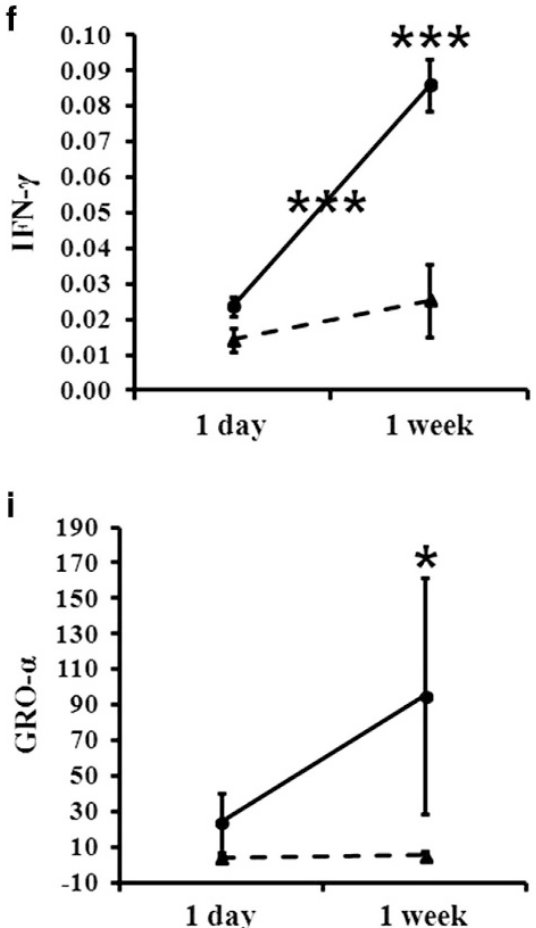

\section{Control - - - - AMSCs}

Figure 4 Measurement of mRNA expression of anti-inflammatory and pro-inflammatory factors after AMSC transplantation. (a-d) mRNA expression of the anti-inflammatory factors CD200, IDO IL-10 and TGF- $\beta$ was higher in the rat brain at 1 week after AMSC transplantation (dashed line) than after control treatment (solid line), as determined via q-PCR, whereas the expression of (e-i) pro-inflammatory factors (IFN- $\gamma$, TNF- $\alpha$, IL-1 $\beta$, MCP-1 and GRO- $\alpha$ ) was lower at 1 week after AMSC transplantation (dashed line) than after control treatment (solid line). ${ }^{* *} P<0.001 ;{ }^{*} P<0.01 ;{ }^{*} P<0.05$ for each comparison.

AMSCs gradually improved the immunomodulatory effects of from 1 day to 1 week in stroke.

Decrease in activated microglia cells after CD200-positive AMSC transplantation in a stroke model

We determined whether the immunomodulatory effects of AMSCs might be involved in CD200 expression after transplantation in vivo by using immunohistochemistry analysis. CD200 expression was significantly higher $(P<0.05)$ in the region transplanted with AMSCs than controls at 1 week after transplantation (Figure 5a). After ischemic brain damage, microglia transform into phagocytes and release a variety of substances, many of which are cytotoxic and/or cytoprotective. An inflammatory response in the brain is associated with changes in microglia shape. Under normal conditions, microglia exhibit a ramified appearance with a large number of processes. In the inflamed brain, microglia acquire a rounded amoeboid shape, and the length and number of processes decreases. To determine the effects of cell transplantation on inflammatory responses, we assessed microglia activation in an acute model of stroke by using Iba-1 staining. The staining intensity of Iba-1-positive cells was measured at 1 week after transplantation (Figure 5b). In the cell treatment group, compared with the PBS control group, the proportion of microglia dispersed in the area significantly decreased, to $50.8 \%$ $(P<0.01)$ after 1 week (Figure $5 \mathrm{~d})$. These results suggested more pronounced attenuation of the inflammatory responses of AMSCs compared with the PBS control group. In addition, 

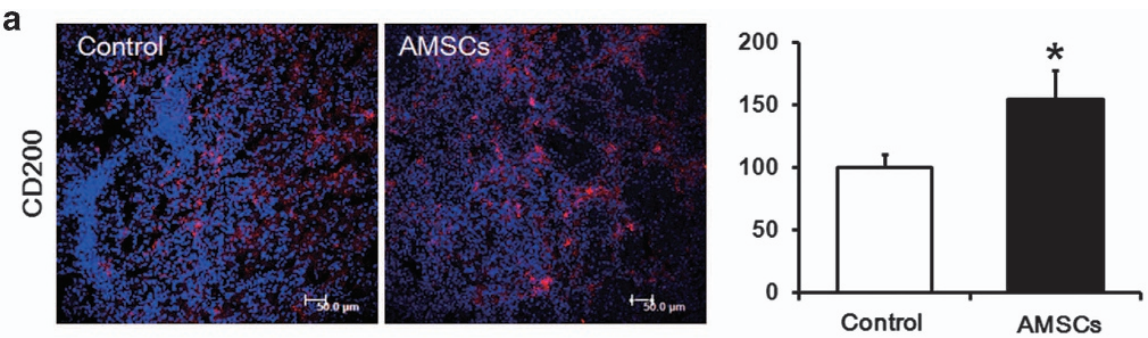

b

Iba1

GFAP/NeuN
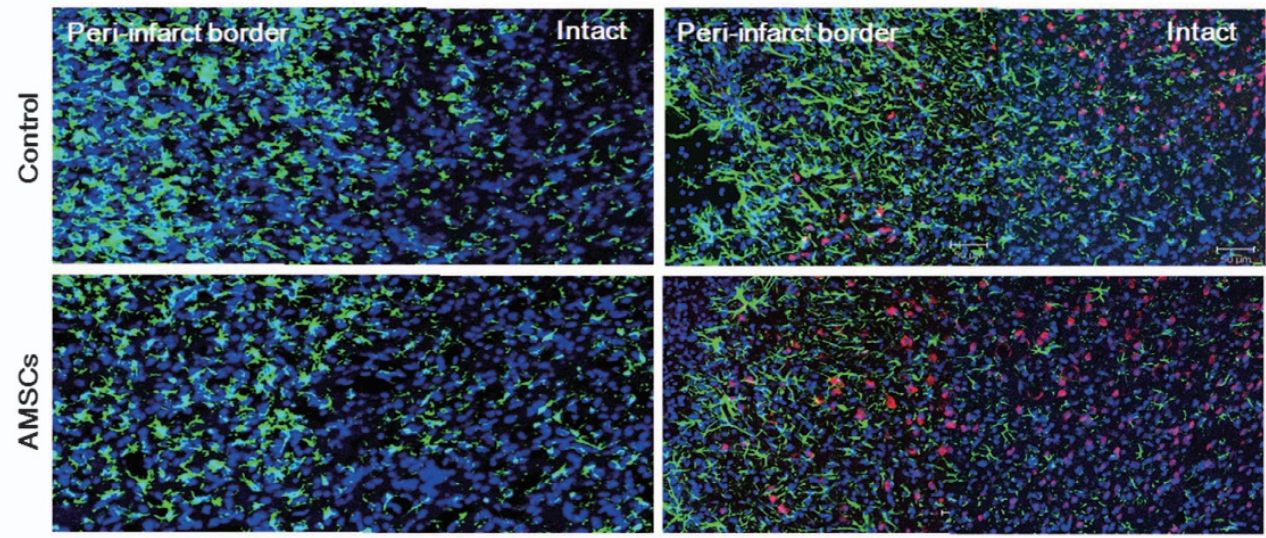

c

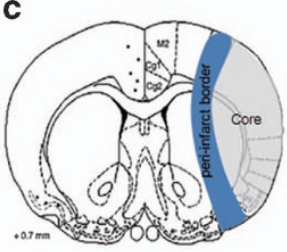

d

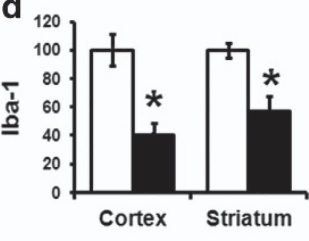

e

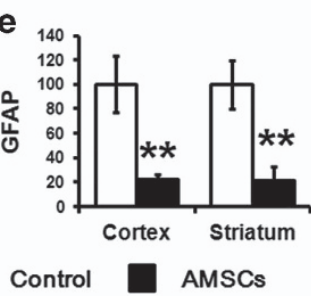

f

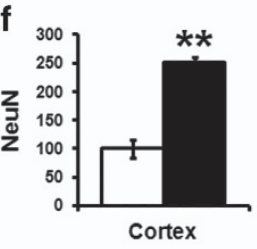

Figure 5 Neuroprotective effect of CD200-positive AMSCs at 1 week after transplantation. (a) CD200 (red) expression merged with DAPI (blue) was significantly higher at 1 week after AMSC transplantation than after control treatment. (b, d) Decreased Iba-1-positive (green) cells merged with DAPI (blue) were detected, particularly in the peri-infarct border, in the AMSC group compared with the control group. (b, e) GFAP-positive cells (green) were also decreased around peri-infarct border, whereas (b, f) NeuN-positive cells (red) were higher in the AMSC group compared with the control group. (c) Schematic diagram of the Iba-1, GFAP and NeuN detection regions. ${ }^{* * *} P<0.001 ;{ }^{* *}$ $P<0.01 ;{ }^{*} P<0.05$ for each comparison. Data are expressed as the mean standard error of the mean. (Scale bar $\left.=50 \mu \mathrm{m}\right)$.

we detected GFAP-positive cells at 1 week after transplantation, and a similar decrease pattern was also observed. The number of GFAP-positive cells dramatically decreased, to approximately $60 \%(P<0.01)$ after AMSC transplantation (Figures $5 \mathrm{~b}$ and $\mathrm{e}$ ). In addition, the group with CD200-positive AMSCs showed a decrease in Iba-1 and GFAP-positive cells compared with the group with CD200-silenced AMSCs (Supplementary Figure S4D). Moreover, numerous NeuN-positive cells were detected in the peri-infarct border region (Figures $5 \mathrm{~b}$ and $\mathrm{f}$ ). A Schematic diagram of rat brain coronal section showed the region of peri-infarct border for the Iba-1, GFAP and NeuN positive cells (Figure $5 \mathrm{c}$ ). These results demonstrated an AMSC-dependent decrease of Iba-1 and GFAP in the early phase of stroke, and these effects subsequently contributed to neuroprotection. Altogether, these results demonstrated that AMSCs are capable of restraining both microglial and astrocytic activation in the early phase of stroke and subsequently protecting neurons against MCAO-related damage.

\section{Effects of CD200-positive AMSC transplantation on the} infarct area and behavioral functional recovery in an MCAO rat model

To investigate the in vivo effect of cell therapy using CD200positive AMSCs, we transplanted AMSCs into an MCAO rat model. The hematoxylin and eosin stained brain tissues of the rats at 6 weeks after transplantation indicated decreased tissue damage in the affected hemisphere. The extent of the damaged area was measured by calculating the ratio of the remaining tissue in the ipsilateral region and the intact tissue in the contralateral region. The transplantation of AMSCs, compared with the control treatment, decreased the extent of brain damage induced by MCAO at 6 weeks (Figure 6a). The proportion of the remaining tissue was significantly higher in the AMSC group $(66.3 \%)$ than in the control group (48\%, $P<0.05$, Figure 6b). Neurological and functional outcomes were measured by using rotarod and mNSS tests before AMSC transplantation, and at 1 day, 1, 2, 3, 4 and 6 weeks after AMSC 
a

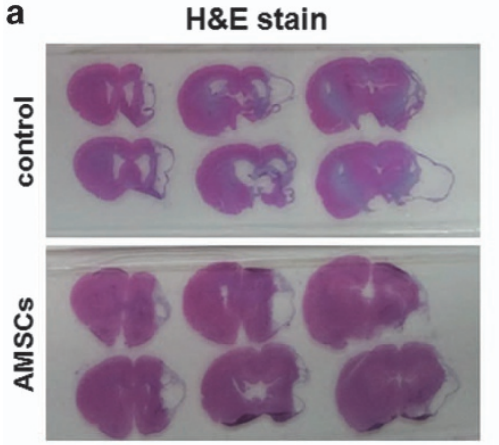

C

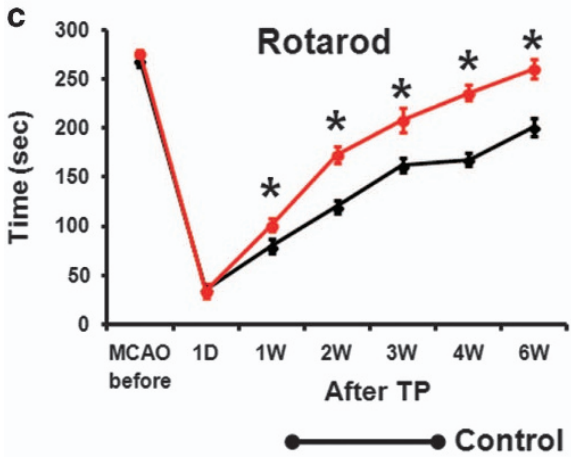

b

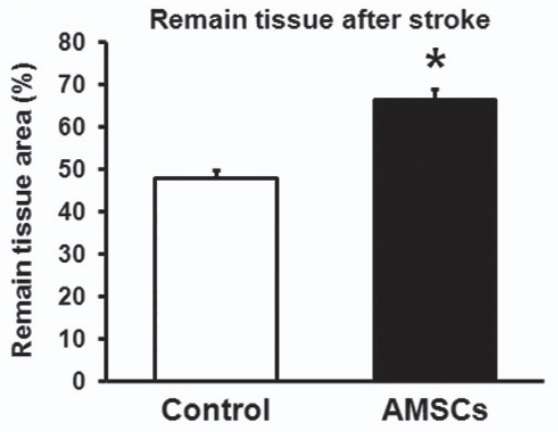

d

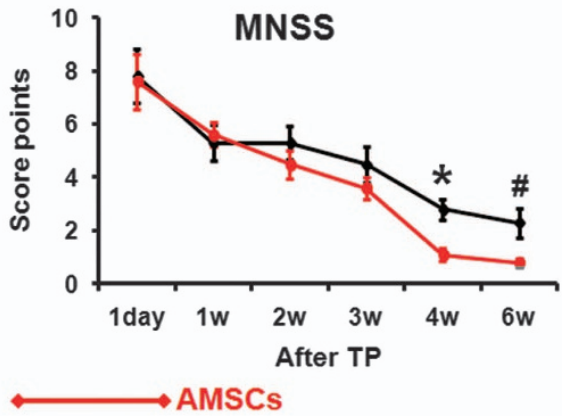

Figure 6 Effects of CD200-positive AMSCs in stroke model after transplantation. (a) Detection of lesion development by using H\&E staining showed distinctly smaller lesion size in the AMSC group 6 weeks after AMSC transplantation (b), characterized by a decrease in the infarct ratio, than in the control group. Behavior test measured in control and AMSC-transplanted groups using (c) rotarod and (d) mNSS tests. Behavior was assessed at 1 day after transplantation and reassessed every week after transplantation. The 6-week AMSCtransplanted group, compared with the control group, showed behavioral improvements in both rotarod and mNSS tests. ${ }^{*} P<0.05$; ${ }^{\#} P<0.06$ for each comparison. Data are expressed as the mean standard error of the mean.

transplantation. On the basis of the results of the rotarod test, significant improvements were observed at 1 week $(P<0.05)$ after transplantation, and these improvements continued through 6 weeks $(P<0.05)$ post infarction (Figure $6 \mathrm{c})$. Similar results were obtained in the mNSS test. Specifically, no significant differences were observed between the AMSC group and the PBS control group in terms of neurological scores immediately after infarction. However, after 4 weeks $(P<0.05)$, the neurological scores were significantly improved in the AMSC group compared with the PBS control group, even at 6 weeks $(P>0.05)$ post infarction (Figure $6 \mathrm{~d})$. Both tests revealed that the apparent functional improvement was timedependent, thus suggesting that as a cell therapy, transplanted AMSCs decrease the infarct size and ameliorate behavioral deficits in MCAO models.

\section{Neuroprotective effect of CD200-positive AMSCs on remaining tissue after transplantation}

We explored whether stroke triggers neurogenesis in both the control and AMSC transplantation groups. To identify the positive effects of CD200-positive AMSCs at 6 weeks after transplantation, the number of Iba-1- and NeuN-positive cells was examined in the remaining brain tissues after MCAO by using immunohistochemistry (Figure 7a). A significantly higher number of NeuN-positive cells $(P<0.05)$ was observed in the AMSC group compared with the control group, whereas the number of Iba-1-positive cells $(P<0.001)$ was significantly lower in the AMSC group than in the control group (Figure 7b). Furthermore, the adoptive transfer of regulatory T-cells (Tregs) showed inflammatory suppression responses in several disease models. ${ }^{41-43}$ To investigate the effects of Tregs in ischemic stroke rat models, we measured the protein expression of Foxp3, CD4 and CD25 in the investigated population of Tregs by using western blotting. The protein levels of Foxp3 were substantially increased in the AMSC group at both 1 and 6 weeks, although no changes in CD4 and CD25 expression were observed (Supplementary Figure 3). Notably, the expression levels of $\mathrm{NeuN}$ and CD200 dramatically increased in the AMSC group (Supplementary Figure 3). Regarding inflammatory responses, AMSC transplantation dramatically decreased the activated form of microglia cells, thereby indicating that cell transplantation suppressed the activity of microglia-induced inflammation. Thus, CD200positive AMSC transplantation represses microglia activation and consequently elicits protective effects on damaged neurons.

\section{DISCUSSION}

Although many types of pathogenesis are related to stroke, a growing number of studies have recently focused on the acute brain inflammation associated with ischemic stroke. Various attempts to control post-stroke inflammation are underway, and MSCs are drawing attention as a new therapeutic agent for 
a
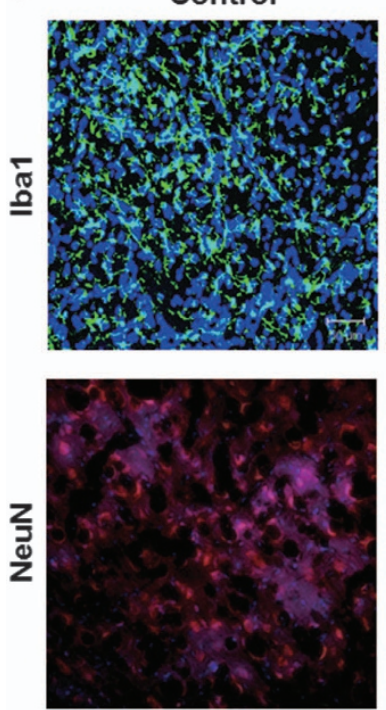

AMSCs
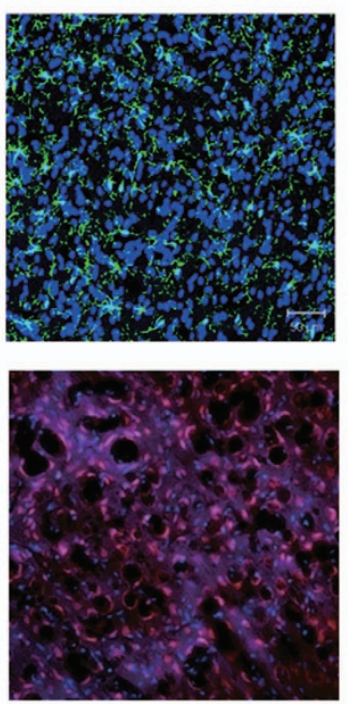

b
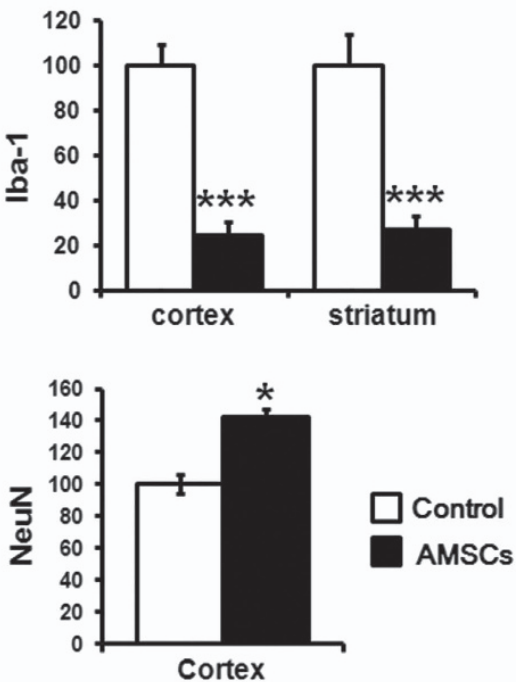

Figure 7 Protective effects of CD200-positive AMSCs on microglia and mature neurons at 6 weeks after transplantation. (a) Decreased Iba1-positive cells and increased NeuN-positive cells were detected at 6 weeks after CD200-positive AMSC transplantation. (b) A significant difference in the number of Iba-1-positive (cortex and striatum) and NeuN-positive cells (cortex) was observed between the AMSCtransplanted group and the control group at 6 weeks after transplantation. ${ }^{* *} P<0.001 ;{ }^{* *} P<0.01 ;{ }^{*} P<0.05$ for each comparison. (Scale bar $=50 \mu \mathrm{m}$ ). Data are expressed as the mean standard error of the mean. (Scale bar $=50 \mu \mathrm{m}$ ).

inflammatory diseases, owing to their unique immunosuppressive ability. Placenta-derived MSCs (PMSCs) exhibit superb immune-suppressive function, as compared with adipo- or bone marrow-derived MSCs. ${ }^{44,45}$, and exert therapeutic effects in neurodegenerative diseases, such as Alzheimer's or Parkinson's disease. ${ }^{24,46}$ In recent studies, the effects of hypoxicconditioned PMSCs on scar formation have been explored. Hypoxic conditions enhance the proliferation of PMSCs and hypoxic-conditioned PMSCs protect against scar formation in vivo. ${ }^{47}$ However, the mechanism by which injected AMSCs survive under barren conditions and elicit therapeutic effects remain to be elucidated. The present study demonstrated that the initial reaction of AMSCs in the ischemic environment through the increased expression of CD200, particularly under hypoxic conditions, played a crucial role in the observed therapeutic effects. The R-seq results (Figure 1) revealed significantly higher CD200 expression under hypoxic conditions compared with normoxic conditions. We further confirmed the role of CD200 among anti-inflammatory factors, thus suggesting that AMSCs expressed higher levels of CD200 compared with other anti-inflammatory factors under ischemic conditions. The siCD200-transfected AMSCs exhibited decreased TGF- $\beta$, IDO and IL-10 expression and introduced a small range of recovery after LPS stimulation. These results further suggested that CD200 may regulate other upstream anti-inflammatory factors, such as TGF- $\beta$, IDO and IL-10. A recent study has reported that the interaction between CD200 and CD200 receptors subsequently induces the expression of anti-inflammatory factors, including IDO, IL-10 and TGF$\beta .^{26,27}$ The results of $\mathrm{q}-\mathrm{PCR}$ analysis showed the increased expression of CD200 and other anti-inflammatory factors, including TGF- $\beta$, IL-10, and IDO, in LPS-stimulated AMSCs.
Thus, we propose that increased CD200 expression induces the targeting of one or more immunosuppressive pathways.

Generally, one to six hours after stroke, microglia cells are activated, and pro-inflammatory cytokines are rapidly upregulated, thereby resulting in inflammatory injuries in the brain. ${ }^{48,49}$ Here we obtained mRNA from the brain at an early stage of stroke in a rat model after cell transplantation. Alterations in cytokine expression at 1 day and 1 week after cell transplantation were measured. At 1 day after transplantation, only CD200 exhibited statistically significant differences between the AMSCs and control groups, whereas other factors exhibited no statistically significant differences. In contrast to the results obtained at 1 day, brain measurements at 1 week post-transplantation revealed a statistically significant decline in all pro-inflammatory cytokines in the AMSC group and a significant increase in immunomodulatory factors. On the basis of this result, we hypothesized that AMSCs may influence innate immune cells in the acute phase in brain tissue, thus relieving brain inflammation in the early stages of a stroke. Moreover, 1 week after AMSC transplantation, a minimal number of cells differentiated into neuronal cells expressing DCX and Tuj1 (Supplementary Figure 2). Tuj1 exhibited imperfect morphology at 1 week after transplantation, and Tuj1 was not detected at 2 week after transplantation (data not shown). This result indicated that after transplantation into the rat brain, the AMSCs regulate inflammatory factors; however, the long-term survival of these cells is poor in the early stages of stroke, thus reflecting the barren environment of the impaired brain region. We hypothesized that the injection of AMSCs in the early stage of stroke controls the disrupted balance of cytokines and favors an environment facilitating recovery. In addition, the ramified morphology of microglia 
after the stroke was altered to an activated morphology, and these cells were observed at high density around the damaged region. After damage, microglia exerted a defensive function in the front line. However, as damage increases over time, reflecting pro-inflammatory cytokine release by activated microglia, the presence of immune modulation factors in an early stage of stroke may provide a crucial therapeutic key to prevent further damage that might lead to a larger infarct volume after stroke. One week after transplantation, the brain contained large amounts of microglia with activated morphology. The intensity of activated microglia in the cell transplantation group was dramatically lower, and more CD200-positive cells were observed in the affected regions, thus consequently leading to long-lasting functional recovery. Recent studies have not only focused on the therapeutic effect of MSC transplantation but also have demonstrated the effects of conditional medium (CM) on MSC-dependent therapy in a stroke model. The CM of bone marrow-derived MSCs has been found to decrease the infarct volume at 1 week post MCAO. ${ }^{50}$ Because the CD200-positive AMSCs exhibited immunomodulatory effects, we assessed whether the CD200 and/or CM expressed in AMSCs might also exert therapeutic effects in a stroke model.

Finally, kinetic functionality was evaluated until the 6th week after transplantation to observe long-term functional improvements. The AMSC group exhibited a statistically significant level of improvement in a time-dependent manner (Figure 6). In relation to this observation, we measured damaged portions in the brain tissue and confirmed a decrease in the sizes of the damaged tissue regions in the AMSC group (Figure 6). On the basis of these results, we hypothesized that the immunomodulatory functions of AMSCs stabilized the activated microglia to a re-ramified resting state in the early stage of stroke under ischemic conditions by controlling the imbalance of cytokines in the acute phase. Then, the converted microglia normalized the impaired toxic condition to a healthy brain environment, thus ultimately improving motor functions and further regenerating damaged neurons. These results indicated the initial effects of high CD200 expression in AMSCs constitute a key therapeutic factor that leads to a healthy brain environment and consequently regenerates neurons, thus potentially facilitating fast recovery. In this study, we used AMSCs to characterize the therapeutic effects of immune regulation in stroke. MSCs can be derived from various tissues, including adipose tissue, peripheral blood, placenta, and bone marrow. Because MSCs from different sources possess different immunomodulatory properties. ${ }^{51}$, further studies should assess whether MSCs derived from other sites also show the increased expression anti-inflammatory factors, particularly CD200, and exhibit an immunomodulatory effect on stroke. To determine how the effects of CD200-positive AMSCs on the immune system are directly linked to the processes underlying neural repair after stroke, we analyzed the short-term immunomodulatory effects of CD200-silenced AMSCs at 1 week after transplantation. To demonstrate the direct and long lasting role of CD200 in AMSCs, more robust knockout experiments are needed. In conclusion, in an environment of inflammatory injuries, AMSCs increase the levels of the immunomodulatory factors, including CD200. This phenomenon is effective in controlling the cytokine imbalance in activated microglia. This function of AMSCs suppresses the expression of pro-inflammatory cytokines produced and decreases microglia activation in the early stages of brain ischemia. The modulating effects of transplanted AMSCs on the innate immune system decrease the inflammatory responses resulting from damage and even provide protective effects, thus ultimately improving motor functions. In conclusion, the present results demonstrated that the MSCs originating in the placenta are an effective cell therapeutic agent for inflammation-related diseases.

\section{CONFLICT OF INTEREST}

The authors declare no conflict of interest.

\section{ACKNOWLEDGEMENTS}

This study was supported by Bio \& Medical Technology Development Program through the National Research Foundation of Korea funded by the Ministry of Science, ICT \& Future Planning (NRF-2017M3A9B4025699 and NRF-2017M3A9B4025709).

1 Schmidt AM, Yan SD, Yan SF, Stern DM. The multiligand receptor RAGE as a progression factor amplifying immune and inflammatory responses. J Clin Invest 2001; 108: 949-955.

2 Jin R, Yang G, Li G. Inflammatory mechanisms in ischemic stroke: role of inflammatory cells. J Leukoc Biol 2010; 87: 779-789.

3 Xie L, Sun F, Wang J, Mao X, Xie L, Yang SH et al. mTOR signaling inhibition modulates macrophage/microglia-mediated neuroinflammation and secondary injury via regulatory T cells after focal ischemia. J Immunol 2014; 192: 6009-6019.

4 Blasko I, Stampfer-Kountchev M, Robatscher P, Veerhuis R, Eikelenboom $P$, Grubeck-Loebenstein B. How chronic inflammation can affect the brain and support the development of Alzheimer's disease in old age: the role of microglia and astrocytes. Aging Cell 2004; 3: 169-176.

5 Patel AR, Ritzel R, McCullough LD, Liu F. Microglia and ischemic stroke: a double-edged sword. Int J Physiol Pathophysiol Pharmacol 2013; 5: 73-90.

6 Ekdahl CT, Claasen JH, Bonde S, Kokaia Z, Lindvall O. Inflammation is detrimental for neurogenesis in adult brain. Proc Natl Acad Sci USA 2003; 100: 13632-13637.

7 Dooley D, Vidal P, Hendrix S. Immunopharmacological intervention for successful neural stem cell therapy: New perspectives in CNS neurogenesis and repair. Pharmacol Ther 2014; 141: 21-31.

$8 \mathrm{Kim}$ YK, Na KS, Myint AM, Leonard BE. The role of pro-inflammatory cytokines in neuroinflammation, neurogenesis and the neuroendocrine system in major depression. Prog Neuropsychopharmacol Biol Psychiatry 2016; 64: 277-284.

9 Parr AM, Tator $\mathrm{CH}$, Keating A. Bone marrow-derived mesenchymal stromal cells for the repair of central nervous system injury. Bone Marrow Transplant 2007; 40: 609-619.

10 Zhang Y, Chopp M, Meng Y, Katakowski M, Xin H, Mahmood A et al. Effect of exosomes derived from multipluripotent mesenchymal stromal cells on functional recovery and neurovascular plasticity in rats after traumatic brain injury. J Neurosurg 2015; 122: 856-867.

11 Calio ML, Marinho DS, Ko GM, Ribeiro RR, Carbonel AF, Oyama LM et al. Transplantation of bone marrow mesenchymal stem cells decreases oxidative stress, apoptosis, and hippocampal damage in brain of a spontaneous stroke model. Free Radic Biol Med 2014; 70: 141-154.

12 Wang Z, Wang Y, Wang Z, Gutkind JS, Wang Z, Wang F et al. Engineered mesenchymal stem cells with enhanced tropism and paracrine secretion of cytokines and growth factors to treat traumatic brain injury. Stem Cells 2015; 33: 456-467.

13 Wang LQ, Lin ZZ, Zhang HX, Shao B, Xiao L, Jiang HG et al. Timing and dose regimens of marrow mesenchymal stem cell transplantation affect the outcomes and neuroinflammatory response after ischemic stroke. CNS Neurosci Ther 2014; 20: 317-326. 
14 Yin F, Guo L, Meng CY, Liu YJ, Lu RF, Li P et al. Transplantation of mesenchymal stem cells exerts anti-apoptotic effects in adult rats after spinal cord ischemia-reperfusion injury. Brain Res 2014; 1561: 1-10.

15 Yoo SW, Kim SS, Lee SY, Lee HS, Kim HS, Lee YD et al. Mesenchymal stem cells promote proliferation of endogenous neural stem cells and survival of newborn cells in a rat stroke model. Exp Mol Med 2008; 40: 387-397.

16 Donega V, Nijboer CH, van Tilborg G, Dijkhuizen RM, Kavelaars A, Heijnen CJ. Intranasally administered mesenchymal stem cells promote a regenerative niche for repair of neonatal ischemic brain injury. Exp Neurol 2014; 261: 53-64.

17 Salgado AJ, Sousa JC, Costa BM, Pires AO, Mateus-Pinheiro A, Teixeira FG et al. Mesenchymal stem cells secretome as a modulator of the neurogenic niche: basic insights and therapeutic opportunities. Front Cell Neurosci 2015; 9: 249.

18 Ooi YY, Ramasamy R, Rahmat Z, Subramaiam H, Tan SW, Abdullah M et al. Bone marrow-derived mesenchymal stem cells modulate BV2 microglia responses to lipopolysaccharide. Int Immunopharmacol 2010; 10: $1532-1540$

19 Uccelli A, de Rosbo NK. The immunomodulatory function of mesenchymal stem cells: mode of action and pathways. Ann NY Acad Sci 2015; 1351: 114-126.

20 Kyurkchiev D, Bochev I, Ivanova-Todorova E, Mourdjeva M, Oreshkova T, Belemezova $\mathrm{K}$ et al. Secretion of immunoregulatory cytokines by mesenchymal stem cells. World J Stem Cells 2014; 6: 552-570.

21 Malcherek G, Jin N, Huckelhoven AG, Mani J, Wang L, Gern U et al. Mesenchymal stromal cells inhibit proliferation of virus-specific CD8(+) T cells. Leukemia 2014; 28: 2388-2394.

22 Gouveia de Andrade AV, Bertolino G, Riewaldt J, Bieback K, Karbanova J, Odendahl $\mathrm{M}$ et al. Extracellular vesicles secreted by bone marrow- and adipose tissue-derived mesenchymal stromal cells fail to suppress lymphocyte proliferation. Stem Cells Dev 2015; 24: 1374-1376.

23 Lee JK, Jin HK, Endo S, Schuchman EH, Carter JE, Bae JS. Intracerebral transplantation of bone marrow-derived mesenchymal stem cells reduces amyloid-beta deposition and rescues memory deficits in Alzheimer's disease mice by modulation of immune responses. Stem Cells 2010; 28: 329-343.

24 Kim KS, Kim HS, Park JM, Kim HW, Park MK, Lee HS et al. Long-term immunomodulatory effect of amniotic stem cells in an Alzheimer's disease model. Neurobiol Aging 2013; 34: 2408-2420.

25 Shi M, Liu ZW, Wang FS. Immunomodulatory properties and therapeutic application of mesenchymal stem cells. Clin Exp Immunol 2011; 164: 1-8.

26 Meuth SG, Simon OJ, Grimm A, Melzer N, Herrmann AM, Spitzer P et al. CNS inflammation and neuronal degeneration is aggravated by impaired CD200-CD200R-mediated macrophage silencing. J Neuroimmunol 2008; 194: 62-69.

27 Holmannova D, Kolackova M, Kondelkova K, Kunes P, Krejsek J, Andrys C. CD200/CD200R paired potent inhibitory molecules regulating immune and inflammatory responses; Part I: CD200/CD200R structure, activation, and function. Acta Medica (Hradec Kralove) 2012; 55: 12-17.

28 Nicholls SM, Copland DA, Vitova A, Kuffova L, Forrester JV, Dick AD. Local targeting of the CD200-CD200R axis does not promote corneal graft survival. Exp Eye Res 2015; 130: 1-8.

29 Kim KS, Park JM, Kong T, Kim C, Bae SH, Kim HW et al. Retinal angiogenesis effects of TGF-betal and paracrine factors secreted from human placental stem cells in response to a pathological environment. Cell Transplant 2016; 25: 1145-1157.

30 Dobin A, Davis CA, Schlesinger F, Drenkow J, Zaleski C, Jha S et al. STAR: ultrafast universal RNA-seq aligner. Bioinformatics 2013; 29: 15-21.

$31 \mathrm{Li} \mathrm{H}$, Handsaker B, Wysoker A, Fennell T, Ruan J, Homer N et al. The sequence alignment/map format and SAMtools. Bioinformatics 2009; 25: 2078-2079.

32 Langmead B, Trapnell C, Pop M, Salzberg SL. Ultrafast and memoryefficient alignment of short DNA sequences to the human genome. Genome Biol 2009; 10: R25.

33 Anders S, Pyl PT, Huber W. HTSeq-a Python framework to work with highthroughput sequencing data. Bioinformatics 2015; 31: 166-169.

34 Anders S, Huber W. Differential expression analysis for sequence count data. Genome Biol 2010; 11: R106.

35 Shannon P, Markiel A, Ozier O, Baliga NS, Wang JT, Ramage D et al. Cytoscape: a software environment for integrated models of biomolecular interaction networks. Genome Res 2003; 13: 2498-2504.
36 Chen J, Li Y, Wang L, Zhang Z, Lu D, Lu M et al. Therapeutic benefit of intravenous administration of bone marrow stromal cells after cerebral ischemia in rats. Stroke 2001; 32: 1005-1011.

37 Abercrombie M. Estimation of nuclear population from microtome sections. Anat Rec 1946; 94: 239-247.

38 Menche J, Sharma A, Kitsak M, Ghiassian SD, Vidal M, Loscalzo J et al. Disease networks. Uncovering disease-disease relationships through the incomplete interactome. Science 2015; 347: 1257601.

39 Vaine CA, Soberman RJ. The CD200-CD200R1 inhibitory signaling pathway: immune regulation and host-pathogen interactions. Adv Immunol 2014; 121: 191-211.

40 Olajide OA, Bhatia HS, de Oliveira AC, Wright CW, Fiebich BL. Inhibition of neuroinflammation in LPS-activated microglia by cryptolepine. Evid Based Complement Alternat Med 2013; 2013: 459723.

41 Reynolds AD, Banerjee R, Liu J, Gendelman HE, Mosley RL. Neuroprotective activities of $\mathrm{CD} 4+\mathrm{CD} 25+$ regulatory $\mathrm{T}$ cells in an animal model of Parkinson's disease. J Leukoc Biol 2007; 82: 1083-1094.

42 Wright GP, Notley CA, Xue SA, Bendle GM, Holler A, Schumacher TN et al. Adoptive therapy with redirected primary regulatory $T$ cells results in antigen-specific suppression of arthritis. Proc Natl Acad Sci USA 2009; 106: 19078-19083.

43 Ishimaru N, Yamada A, Kohashi M, Arakaki R, Takahashi T, Izumi K et al. Development of inflammatory bowel disease in Long-Evans Cinnamon rats based on $\mathrm{CD} 4+\mathrm{CD} 25+\mathrm{Foxp} 3+$ regulatory $\mathrm{T}$ cell dysfunction. J Immunol 2008; 180: 6997-7008.

44 Hass R, Kasper C, Bohm S, Jacobs R. Different populations and sources of human mesenchymal stem cells (MSC): a comparison of adult and neonatal tissue-derived MSC. Cell Commun Signal 2011; 9: 12.

45 Lee JM, Jung J, Lee HJ, Jeong SJ, Cho KJ, Hwang SG et al. Comparison of immunomodulatory effects of placenta mesenchymal stem cells with bone marrow and adipose mesenchymal stem cells. Int Immunopharmacol 2012; 13: 219-224.

46 Kim EY, Lee KB, Kim MK. The potential of mesenchymal stem cells derived from amniotic membrane and amniotic fluid for neuronal regenerative therapy. BMB Rep 2014; 47: 135-140.

47 Du L, Lv R, Yang X, Cheng S, Ma T, Xu J. Hypoxic conditioned medium of placenta-derived mesenchymal stem cells protects against scar formation. Life Sci 2016; 149: 51-57.

48 Ceulemans AG, Zgavc T, Kooijman R, Hachimi-Idrissi S, Sarre S, Michotte $\mathrm{Y}$. The dual role of the neuroinflammatory response after ischemic stroke: modulatory effects of hypothermia. J Neuroinflammation 2010; 7: 74.

49 Lakhan SE, Kirchgessner A, Hofer M. Inflammatory mechanisms in ischemic stroke: therapeutic approaches. J Trans/ Med 2009; 7: 97.

50 Tsai MJ, Tsai SK, Hu BR, Liou DY, Huang SL, Huang MC et al. Recovery of neurological function of ischemic stroke by application of conditioned medium of bone marrow mesenchymal stem cells derived from normal and cerebral ischemia rats. J Biomed Sci 2014; 21: 5.

51 Collins E, Gu F, Qi M, Molano I, Ruiz P, Sun L et al. Differential efficacy of human mesenchymal stem cells based on source of origin. J Immunol 2014; 193: 4381-4390.

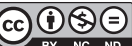

This work is licensed under a Creative Commons Attribution-NonCommercial-NoDerivs 4.0 International License. The images or other third party material in this article are included in the article's Creative Commons license, unless indicated otherwise in the credit line; if the material is not included under the Creative Commons license, users will need to obtain permission from the license holder to reproduce the material. To view a copy of this license, visit http://creativecommons.org/licenses/by-nc-nd/4.0/

(C) The Author(s) 2018

Supplementary Information accompanies the paper on Experimental \& Molecular Medicine website (http://www.nature.com/emm) 